\title{
An Enhanced Method for Web Ontologies Encryption
}

\author{
Redouane Nejjahi, Abderrahim Marzouk, and Noreddine Gherabi
}

\begin{abstract}
Ontologies are used in almost any domain; they have been used both in the public and private domains. In the latter case, we will face security problems, where encryption may be a solution. In this case, one can encrypt their ontology file with any encryption standard, delete the original file and store the encrypted one. When exploiting the ontology, the user should decrypt the encrypted file on the fly and make the needed reasoning. In this paper, we introduce an enhanced method for encrypting web ontologies. This method consists of maintaining the ontology structure (schema) while encrypting only the ontology data. We found that the enhanced method is at least ten times faster than the basic method. With such results, we believe that existing tools for querying or making reasoning on ontologies can be rewritten to take advantage of this method in the case of ontology security.
\end{abstract}

Index Terms-AES; Cryptography; Ontology; OWL; Semantic Web; Security.

\section{INTRODUCTION}

Over the past decade, the web has expanded exponentially [1]. One of its new key features is the semantic web [2]. In this kind of web, ontologies are regarded as its cornerstone; they have been developed in biology, biomedicine, linguistics, entertainment, etc. Specialists from almost every domain have developed their ontologies, either in the public domain or the private one. When working on private ontologies developed for private uses where data is sensitive, we face security issues that we can resolve by encrypting the ontology file [3]. Such encrypted file is useless until it is decrypted where data comes back into its structured state. To do so, people encrypt their ontologies files and store them while deleting the plain text files. When they want to process an ontology, they should first decrypt the entire file.

In this paper, we present an enhanced method to encrypt ontologies. This method consists of separating the structure of the ontology from its content. It seems to be obvious that the content of the ontology (such as the content of individuals) is what we need to hide while the structure presents no sensitive information. Thus, the approach of this method is to encrypt the sensitive data of the ontology while keeping its structure (schema) untouched; the result is a file containing the encrypted data and a file containing the same ontology structure with, for each individual, pointers to the place (offset) in the encrypted file where the encrypted individual's content is written.

\section{TyPeS OF ONTOLOGIES}

OWL Ontologies contain several parts. In the most important parts we have classes, individuals and properties. Classes and properties describe the structure of the ontology where individuals represent its content [4]. Such kind of ontologies applies for example to the FOAF (Friend of a Friend) ontology where classes and properties describe a person, his activities and his relations to other people. This part of the ontology is almost static. Another component of the FOAF ontology is the individuals that we populate the ontology with. This part is dynamic and grows in size exponentially until it occupies almost the entire size of the ontology. Another kind of ontologies is that where there are no individuals and a class does not represent a model of individuals but contains itself the data presented by the ontology. Such kind of ontologies include the Gene Ontology (GO) [5], an ontology of the biological area. In [6], the authors used this classification to reduce the size of ontologies by extracting only segments that correspond to a criterion.

In both kinds of ontologies, we will only secure the data whether it is contained in individuals or directly in the classes.

\section{EXAMPLE ONTOLOGIES}

In this paper, we use, for the explanation purpose, as an example ontology the FOAF (Friend of a Friend) ontology. As for the test part, we use the Gene Ontology (GO) which is $148 \mathrm{MB}$ in size.

\section{ENCRYPTING AND DECRYPTING TEXTS}

The four major goals of cryptography are confidentiality, integrity, authentication, and nonrepudiation [3]. In this paper, we are interested in data confidentiality which is guaranteed by using encryption techniques. An encryption technique is a process that takes a plaintext as input and outputs a ciphertext, a text that is unreadable by third parties who do not hold the key of decryption. The couple of encryption and decryption algorithms is called a cipher. Many ciphers have been developed to ensure data confidentiality. All the new ciphers can be categorized into two categories, symmetric and asymmetric algorithms. Symmetric algorithms use the same key to encrypt and decrypt texts, where asymmetric algorithms use two different keys to encrypt and decrypt texts, public key and private key respectively. It is easy to generate the public key from the private one, but it should be impossible to get the private key given the public one. The symmetric algorithms are faster than the asymmetric ones, but they have the problem to share the key between two communicating entities. If the key, being sent on the network, is intercepted by third parties, then they can decrypt the encrypted messages. In our case, the user encrypts the ontology 
content to prevent its exploitation by anyone else even if they get access to the file. The user decrypts the ontology back to its initial format each time they want to make reasoning on it, and this only the time of the processing. In this case, it's the user who will encrypt and decrypt the texts, which avoids the problem of sharing the key. Therefore, our choice is symmetric algorithms which are faster. We chose to use the Advanced Encryption Standard AES [7], one of the most used and powerful symmetric algorithms [8]. AES can be used with three different key lengths: 128, 192 and 256 bits. In our case, we will use AES with a key length of 128 bits.

\section{The Basic Method For Web Ontologies ENCRYPTION}

The basic method consists of encrypting the entire ontology file. The output is an encrypted file which is useless unless it is decrypted. This step is done only ounce, unless the content of the ontology has changed. Whenever we want to process the ontology in question, we should start by decrypting the entire file to restore the ontology, and then do the process.

\section{OUR ENHANCED METHOD FOR Web ONTOLOGIES ENCRYPTION}

As described above in types of ontologies, we differentiate between two kinds, one that uses classes as models for individuals and another where each class represents in itself a unique individual. Our method consists of leaving the structure of the ontology and all the metadata untouched since this information seems to have no security issues and thus can be left plaintext. As regards data concerning individuals like a person's friends, hobbies, activities, etc., the method encrypts them using AES algorithm. Thus, given an ontology file to encrypt, we can generate a file containing the same general data (like annotation properties, classes definitions, individuals' tags, etc.) in plaintext format with all the data to hide (like individuals' contents) in ciphertext format.

Fig. 1 shows the original ontology file and the output encrypted file.
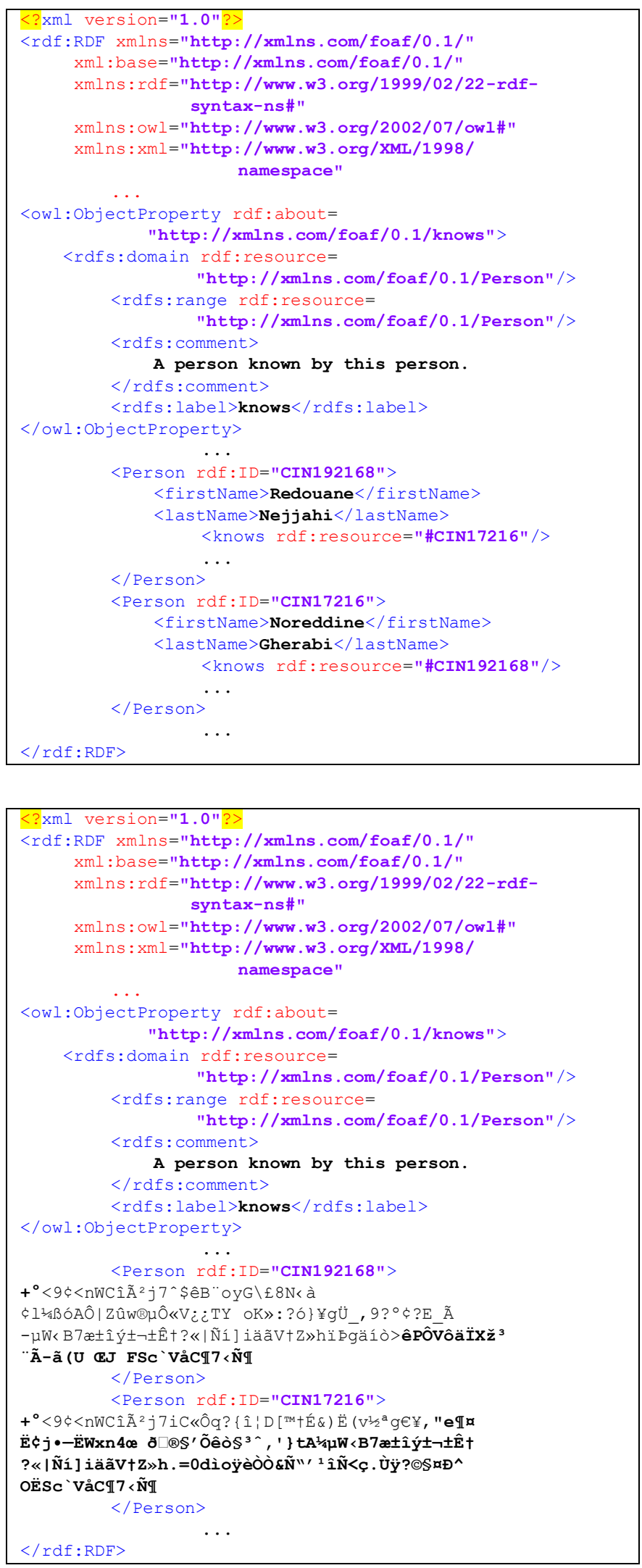

Fig. 1: Original ontology file and output encrypted file.

As we can see, an individual definition like this:

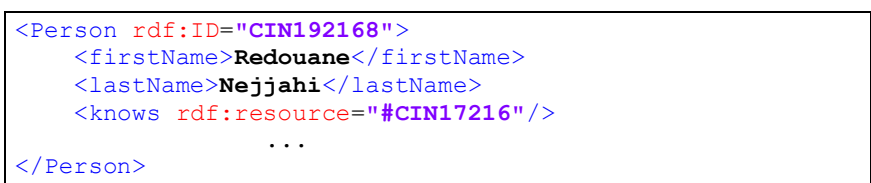


is transformed to something like this:

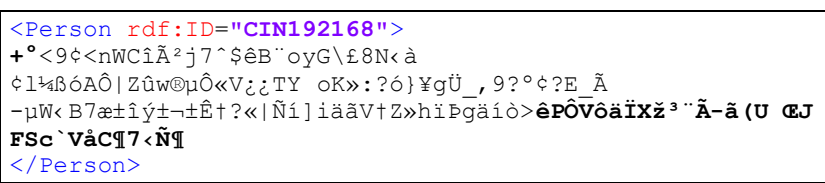

We keep the individual opening and closing tags untouched and we encrypt its content.

The problem with this method is that the generated file is no longer an OWL valid file. Hence, we cannot use the API tools that are based on OWL parsing. Even if we develop our tools, we cannot guarantee that the encrypted content does not contain some characters that are OWL tags. Therefore, the solution that we adopted is to put the encrypted data into a separate file and to put in the ontology file, between each individual's tags, two integer values: the first contains the size of the encrypted data and the second one the offset of this data in the encrypted file. We could use only one integer value, either the data size or its offset in the encrypted file, but when we will try to restore the plaintext of that encrypted data we will be led to do an extra work to determine where the data begins and where it ends in the encrypted file.

Thus, given an ontology file in plaintext format, our enhanced method will generate two files, one containing the same ontology file except the private data which is replaced by two integers with no impact on the security of the ontology and an encrypted file containing the sensitive data in ciphertext format

We call the first file a semi-ontology file, because, on the one hand, it is a valid ontology file and, on the other hand, it has no interesting data as an ontology is supposed to have.

A part of the semi-ontology file is shown below.

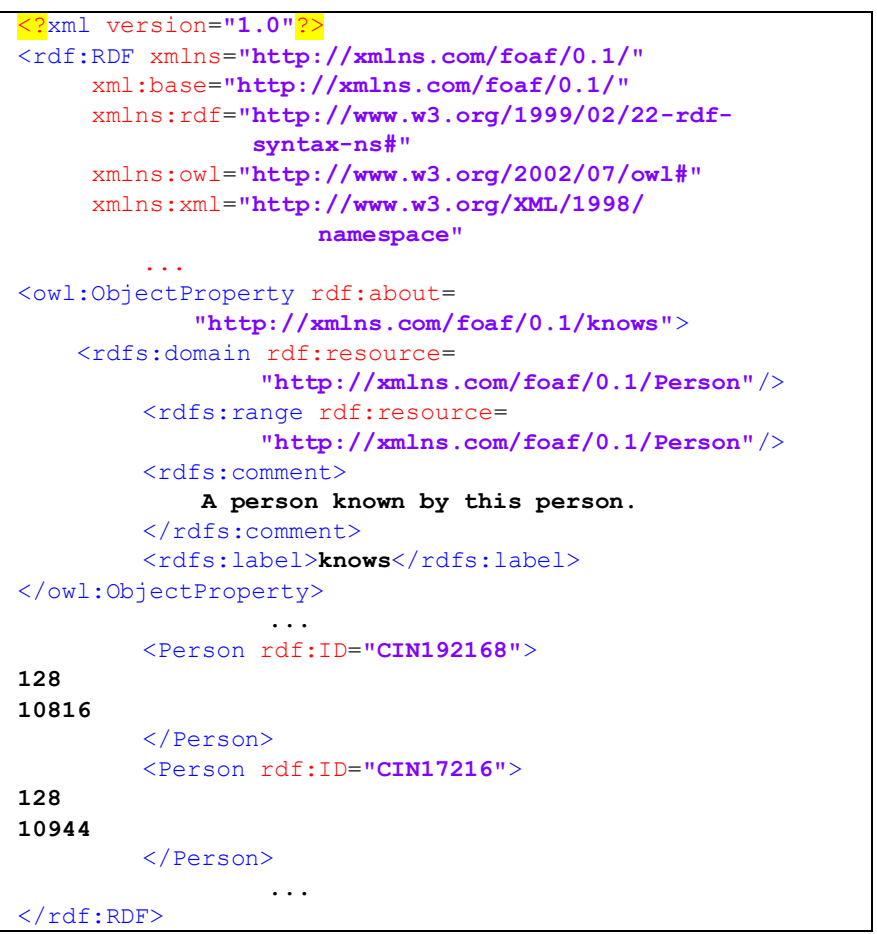

Fig. 2: An excerpt of the semi-ontology file.

The algorithm of our enhanced method is as follows:

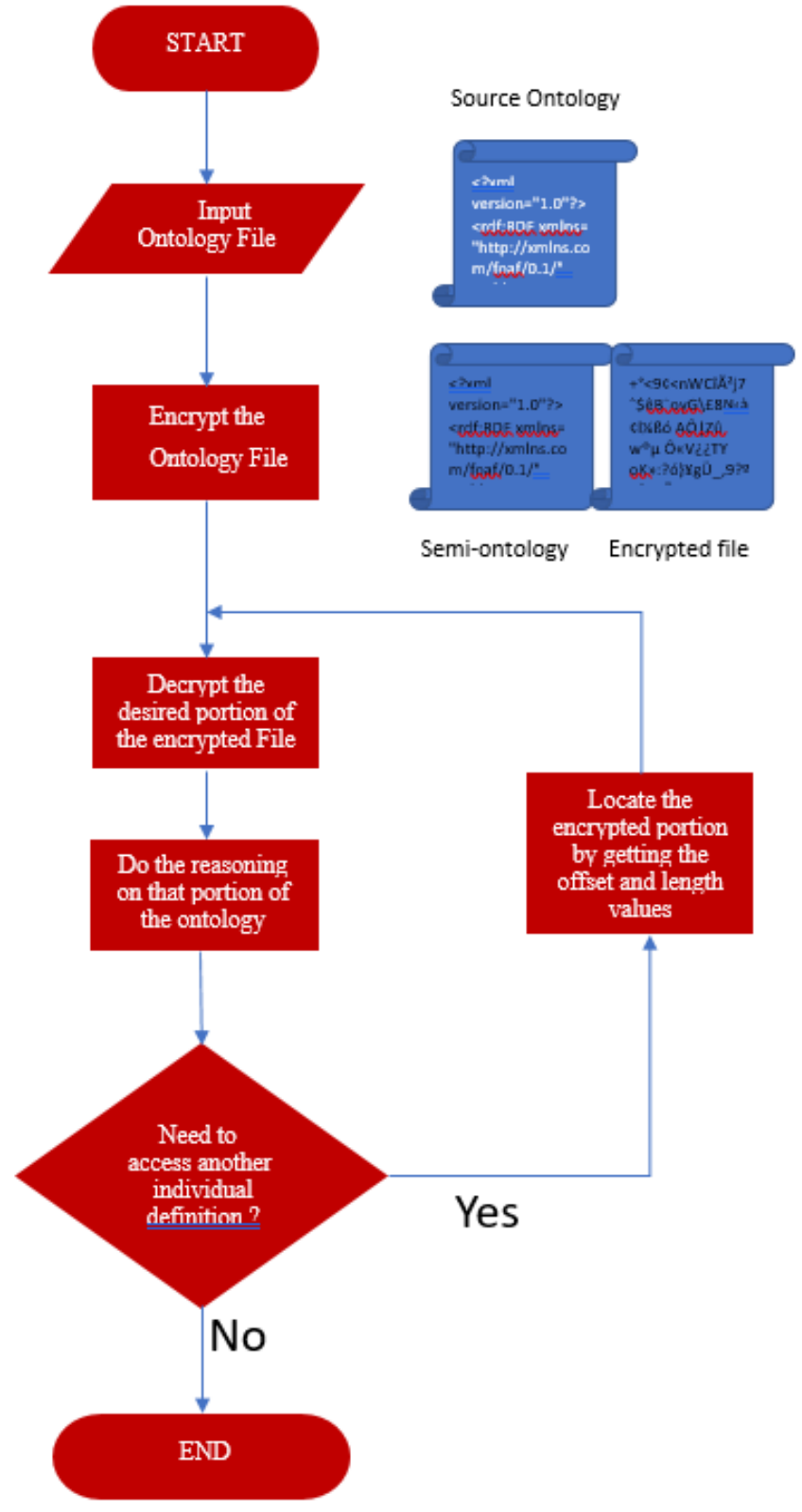

Fig. 3: Flowchart of the enhanced method algorithm.

\section{First step:}

The input Ontology file is transformed to two files: the first one represents the same ontology file except the private data which is replaced by two integers with no impact on the security (a semi-ontology file) and the second one is an encrypted file which contains the sensitive data in ciphertext format.

\section{Next step (each time we need to process the ontology):}

Given two files: Semi-ontology (Plaintext) and the encrypted file, we start the process on the plaintext (the semi-ontology file), then we determine the opening and closing tags of the individual or the class in question, we extract the two integers between the tags which determines where the encrypted portion of data is located in the encrypted file. Finally, we decrypt the extracted data from the encrypted file. On this portion of data which is in plaintext we can apply any desired treatments. If we need to access another individual, we repeat this last step one more 
time to extract the two integers, localize the desired portion in the encrypted file and then decrypt this portion of data; and so on.

\section{Evaluation}

To evaluate our enhanced method compared to the basic one, we measured the time made by each method, in several moments and with different resources, to process the ontology. We suppose that the first step, i.e. the encryption of the entire file for the basic method and the generation of the semi-ontology file with the encrypted private data file for our enhanced method, has already been done. The results are shown in Fig. 4.

\section{Time made by each method}

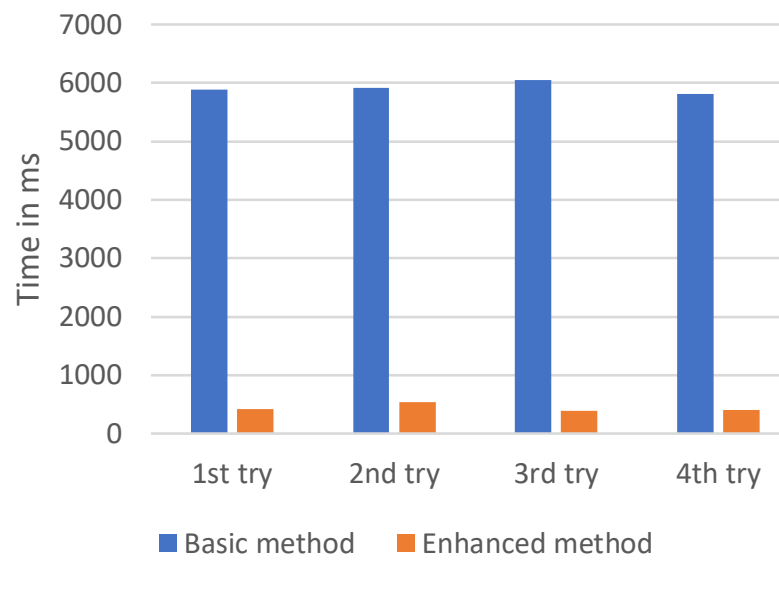

Fig. 4: Time of execution in milliseconds for Basic and Enhanced methods.

As we can see in this figure, the enhanced method is at least ten times faster than the basic one.

\section{FUTURE WORK}

We hope that new tools for querying and making reasoning on ontologies where security is a primer issue can be made based on the existent ones to take advantage of our enhanced method.

As a future work, we will examine our method in the field of ontologies compression.

\section{CONCLUSION}

When working with private ontologies that contain sensitive information, some issues appear, like securing the ontology files residing in the local machine. Instead of securing the network from cyber-attacks, which requires a lot of resources and even does not protect data if the machine was locally accessed by a third party, we can rely on files encryption. Without the key to decrypt the files, they are useless. Before making any reasoning on the ontology, it must be decrypted first.

In our enhanced method that we presented in this paper, we transformed the ontology file (in plaintext) to two files. One is a valid ontology file in plaintext format but with no sensitive data and the other one is a ciphertext file containing the encrypted sensitive data. The benefit of this method is that we can make reasoning on the first file which is a valid ontology, and if we need to get access to an individual data, we extract just the corresponding portion from the second file and we decrypt it. Since we decrypt only the desired information, our method gains a lot of time especially when dealing with big ontologies.

\section{REFERENCES}

[1] S. Aghaei, M. A. Nematbakhsh, and H. K. Farsani, "Evolution of the World Wide Web: from Web 1.0 to Web 4.0.", International Journal of Web \& Semantic Technology (IJWesT) Vol.3, No.1, January 2012.

[2] T. Berners-Lee, J. Hendler, and O. Lassila, "The semantic Web", Scientific American, vol. 284, no. 5, pp. 34-43, May 2001.

[3] A. J. Menezes, P. C. van Oorschot, S. A. Vanstone, Handbook of Applied Cryptography, $1^{\text {st }}$ edition, CRC Press, 1997.

[4] S. Bechhofer. "OWL: Web Ontology Language," in Encyclopedia of Database Systems, ed. Springer, 2009.

[5] B. Smith, J. Williams, and S-K. Steffen, "The ontology of the Gene Ontology", AMIA Annual Symposium proceedings, pp. 609-613, 2003.

[6] R. Nejjahi, N. Gherabi, and A. Marzouk, "Towards classification of Web ontologies using the horizontal and vertical segmentation", Advances in Intelligent Systems and Computing, vol. 640, pp. 70-81, DOI 10.1007/978-3-319-64719-7, Springer International Publishing 2017.

[7] J. Daemen and V Rijmen. "The design of Rijndael: AES - The Advanced Encryption Standard (Information Security and Cryptography)", Springer, 1st ed. 2002.

[8] D. S. Abd Elminaam, H. M. Abdual Kader, and M. M. Hadhoud, "Evaluating the performance of symmetric encryption algorithms", International Journal of Network Security, Vol.10, No.3, pp. 213-219, May 2010.

Redouane Nejjahi received his B.S. degree in Information Technology from the Faculty of Science and Technology, Settat, Morocco, in 2002, and the M.S. degree in Informatics and Telecommunication from the Faculty of Science, Rabat, Morocco, in 2005. Currently, he prepares his Ph.D. in Information Technology at the IR2M Laboratory in the Faculty of Science and Technology, Settat, Morocco.

Abderrahim Marzouk received his $\mathrm{PhD}$ in Computer Science from University of Caen, France, in 1995. He has more than 15 years of experience in teaching Computer Science, JEE Technology and Web Applications. His current research interests are Software Engineering, Software Security and Software Process Modeling. Mr. Marzouk is a full professor at the Faculty of Science and Technology, Settat, Morocco.

Noreddine Gherabi received his B.S. degree in Information Technology, M.S. and Ph.D. from the Faculty of Science and Technology, Settat, Morocco. Currently, he is a full professor at the National School of Applied Sciences, Khouribga, Morocco. 